Correo: innova@uide.edu.ec

\title{
Arte y lectura: vínculos entre universidad y comunidad
}

\section{Art and reading: links between the university and the community}

\author{
Dra. Keila Ketty Herrera Rivas \\ Mgs. Rosa Armijos Acosta \\ Universidad de Guayaquil, Ecuador
}

Autor para correspondencia: rosa.armijosa@ug.edu.ec, keila.herrerar@ug.edu.ec

Fecha de recepción: 01 de Septiembre de 2016 - Fecha de aceptación: 01 de Noviembre de 2016

\section{Resumen}

El vínculo universidad - sociedad actualmente constituye uno de los factores más sensibles a la hora de valorar y apreciar la pertinencia de la universidad y su entorno, en la actualidad la educación superior del Ecuador asumió el mandato de que la universidad es la encargada, en mayor grado, de preservar, crear, desarrollar y difundir la cultura de la humanidad. El papel que cumple y el lugar que ocupan las instituciones de educación superior en función de las necesidades y demandas de los diversos sectores sociales. Las acciones que se formulen carecerán de real sentido social si no son anticipatorias de escenarios futuros y no manifiesten su intención de modificar la realidad vigente" (UNESCO, 2007), En tal sentido se mantiene un estrecho vínculo con la sociedad, generando programas y proyectos que respondan a las necesidades de determinadas entidades y comunidades ecuatorianas; desarrollar habilidades, fomentar el hábito lector y potenciar el desarrollo de la creatividad artística se presentan como factores determinantes en una sociedad que busca cambios estructurales, actividades que influyen en el desarrollo del pensamiento creativo, crítico; ejes necesarios para vincular el arte y la lectura como estrategias de aprendizaje para el mejoramiento académico.

Palabras claves: vínculo; universidad; comunidad; arte y lectura

\begin{abstract}
The link between university and community is one of the most sensible factor at the momento for valuation and apreciation of the relevance of college and the environment. Nowadays the superior education of Ecuador has assumed the responsability of preservation, creation, development and promotion of the culture. According to the UNESCO, the roll they play is according the needs and demands of the society. Their efforts and plans will have no senseif they do not anticipate future scenearies and show how they want to change the reaility. (UNESCO, 2007). From that perspective and the narrow relationship between community and university it is important to increase reading hobbies, in order to develop creative thinkers, critics who will have the opportunity to use the Reading as an estrategie of learning.
\end{abstract}

Key words: link; university; community; art and reading 


\section{Introducción}

El vínculo universidad - sociedad actualmente constituye uno de los factores más sensibles a la hora de valorar y apreciar la pertinencia de la universidad y su entorno, La UNESCO, en la Conferencia Regional sobre Políticas y Estrategias para la Transformación de la Educación Superior en América Latina y El Caribe al referirse a la pertinencia de la universidad señala lo siguiente:

“[...] el papel que cumple y el lugar que ocupa la educación superior en función de las necesidades y demandas de los diversos sectores sociales. Las acciones que se formulen carecerán de real sentido social si no son anticipatorias de escenarios futuros y no manifiesten su intención de modificar la realidad vigente" (UNESCO, 2007).

En la 5ta Conferencia Internacional de Educación para Personas Adultas (CONFINTEA V), se expresó que la educación sólo tendrá éxito en la sociedad si se genera un vínculo entre los factores del entorno que impulsan las acciones de los individuos, quienes tienen como meta la mejora de la calidad de vida. Entre las variables a considerar están las realidades socio-culturales y económicas, como ejes principales del desarrollo. (UNESCO, 1997)

En la actualidad la educación superior del Ecuador, asumió y se empoderé la idea de que la universidad es la encargada, en mayor grado de preservar, crear, desarrollar y difundir la cultura de la humanidad. En tal sentido se mantiene un estrecho vínculo con la sociedad, generando programas y proyectos que responden a las necesidades de determinadas entidades y comunidades ecuatorianas.

Un estudio realizado en el año 2014, por el Centro Regional para el Fomento del Libro en América Latina y el Caribe (CERLALC) en conjunto con la UNESCO, dio a conocer el índice de lectura a nivel latinoamericano, en donde se determinó que en el Ecuador cada persona lee 0,5 libros al año. (CERLALC, 2015). Se procedió a realizar un estudio en la comunidad Cerrito de los Morreños, en donde a través de la observación y de un diagnóstico in situ se pudo determinar el bajo interés por la lectura en niños, jóvenes y moradores de la comunidad, debido a que no se ha cultivado el hábito lector ni el amor por la lectura, sumado a esto, no cuentan con una biblioteca; los estudiantes de la comunidad necesitan desarrollar habilidades, fomentar el hábito lector y potenciar el desarrollo de la creatividad artística.

Arte y Lectura se presentan como factores determinantes en una sociedad que busca cambios estructurales, de conformidad a los resultados obtenidos en la investigación realizada a los estudiantes de la comunidad Cerrito de los Morreños, se ha considerado al arte como componente básico de una educación integral que permite al individuo desarrollarse plenamente. Por ello se considera a la UNESCO, que manifiesta que toda persona tiene derecho a tomar parte libremente en la vida cultural de la comunidad, a gozar de las artes y a participar en el progreso científico y en los beneficios que de él resulten". (UNESCO, 2006). Gracias al estudio realizado y a la revisión de literatura se pudo determinar que el arte puede llegar a ser no solamente un modo de expresión sino una herramienta para vincular a la universidad con la comunidad, de tal modo que se pueda fomentar un hábito lector a través del aprendizaje empírico, aprender haciendo. 
Por ende se buscan actividades que brinden la oportunidad a niños y niñas de ser partícipes de un proceso creativo, y en este intervienen sentimientos y emociones que hacen única la experiencia ya que forma parte de lo que se está creando, 'Según las investigaciones realizadas por la Naciones Unidas, cuando una persona en fase de aprendizaje entra en contacto con procesos artísticos y recibe una enseñanza que incorpora elementos de su propia cultura, esto estimula su creatividad, su iniciativa, su imaginación, su inteligencia emocional y, además , le dota de una orientación moral”. (UNESCO, 2006)

Una educación artística según un informe de las Naciones Unidas tiene importancia en el desarrollo de una sociedad creativa y sensibilizada a la cultura, por tal motivo esta no solo sirve para crear objetos bonitos y/o poderlos apreciar, sino que este sirve para potenciar el aprendizaje de cualquier tema, esta enseñanza puede aplicarse a pequeños grupos dotados de habilidades o en personas que incursionan por gusto, curiosidad sin necesidad que dominen una técnica.

(UNESCO, 2012)

En lo referente a las artes en todos sus campos han surgido significativos avances en lo que respecta a la valoración crítica y cómo esta influye en la percepción estética del individuo. Lo artístico y lo estético está íntimamente relacionado con la lectura; lo estético, nos permite a través del arte comunicar, es imposible suprimir su carácter expresivo, dado por su nivel pragmático, semántico y sintáctico.

A través de la observación y de un diagnóstico se ha podido determinar el bajo interés por la lectura en niños, jóvenes, padres, madres de familia y moradores de la comunidad "Cerrito de los Morreños" debido a que no se ha cultivado el hábito lector ni el amor por la lectura, sumado a esto que no cuentan con una biblioteca y el desconocimiento de las actividades artísticas y culturales influyen en el desarrollo del pensamiento creativo, crítico; ejes necesarios para vincular el arte y la lectura como estrategias de aprendizaje para el mejoramiento académico en esta comunidad.

Es un plan formativo y experimental, centrado en el paradigma socio crítico que tiene como objetivo aplicar una estrategia metodológica que estimule la imaginación, el pensamiento lógico crítico y creativo en los estudiantes de la comunidad a través del arte y la lectura, porque permiten abrir espacios para mejorar la influencia de acciones transferidas por la acción social de la persona en la forma en que se aprende las conductas sociales y como la interacción influye y mejora el aprendizaje académico.

Estrategia metodológica, centrado en el trabajo productivo, utiliza didáctica como una estrategia dinamizadora del aprendizaje, el docente es mediador, animador, 1 fundamentado por la metodología, acción, reflexión y acción, que asume como estructura el escenario donde se desarrolla el individuo, basado en la creación y experiencias., se propone:

- Que el niño/a. joven, adulto no lector, descubra el libro

- Ayudar a pasar de la Lectura pasiva a la lectura activa 
- Desarrollar el niño/a. joven, adulto el placer de leer

- Relacionar la vivencia emocional, la fantasía y la imaginación

- Estimular la capacidad de apreciación (observar, comparar, sentir, expresar, valorar).

Para que puedan comprender, gozar, además esta vinculación del arte con la lectura permita comprender y disfrutar para luego reflexionar. Este programa está dirigido a estudiantes comprendidos entre 5 y 17 años de edad, padres y madres de familia y moradores entre 15 y 50 años o más, por ello, Diseñar una estrategia metodológica para contribuir a la promoción de la lectura que estimule la imaginación, el pensamiento lógico, crítico y creativo de los estudiantes de esta comunidad del Golfo de Guayaquil.

\section{Bibliografía}

García, A. S. (2015). Enseñanza y aprendizaje en la educación artística. ISSN: 17948614.

CERLALC (2015), 'El libro en cifras', Boletín estadístico del libro en Iberoamérica, volumen No. 7, ISSN 2322-6234.

David Perkins "La escuela inteligente" del adiestramiento de la memoria a la educación de la mente Barcelona, 1997 editorial GEDISA

Eisner W. Elliot. (1995) Educar la visión artística. Paidos Educador. Barcelona, España

Francesco de Bartolomeis "El color de los pensamientos y de los sentimientos", España 2001, Ediciones Octaedro.

Herrera, H. (1995), La comunidad y su Estudio, página 134, Editorial Pueblo y Educación, La Habana, Cuba.

Gardner, Howard (2011), “Las cinco mentes del futuro” Barcelona, Buenos Aires, México Paidós.

Gardner, Howard (2011), “Arte, mente y cerebro" una aproximación cognitiva a la creatividad, Barcelona, Buenos Aires, México Paidós.

Proveyer C. (2000), Selección de lecturas de Trabajo Social Comunitario/Clotilde Proveyer Cervantes, página 168, Curso de formación de trabajadores sociales, La Habana, Cuba.

UNESCO (1997), ''Las universidades y la educación'”, Confintea V, Hamburgo. Documento del sitio web http://www.unesco.org/education/uie/confintea/pdf/2a_span.pdf

UNESCO (2012), 'Educación artística'. Obtenido del sitio web http://www.unesco.org/new/es/culture/themes/creativity/arts-education/ 
UNESCO (2013), ''El fomento a la lectura será parte clave de la Agenda Educacional Post 2015'. Obtenido del sitio web http://portal.unesco.org/geography/es/ev.phpURL_ID=16807\&URL_DO=TOPIC\&URL_SECTION=201.html 